\title{
Comparison of Patient-Reported Outcomes at 1 Year After Injury Between Limb Salvage and Amputation: A Prospective Cohort Study
}

Taketo Kurozumi ( $\boldsymbol{\nabla}$ taketo_kurozumi@m6.dion.ne.jp)

Teikyo University: Teikyo Daigaku https://orcid.org/0000-0003-0270-7993

Takahiro Inui

Teikyo University: Teikyo Daigaku

Yuhei Nakayama

Teikyo University: Teikyo Daigaku

Akifumi Honda

Teikyo University: Teikyo Daigaku

Kentaro Matsui

Teikyo University: Teikyo Daigaku

Keisuke Ishii

Teikyo University: Teikyo Daigaku

Takashi Suzuki

Teikyo University: Teikyo Daigaku

Yoshinobu Watanabe

Teikyo University: Teikyo Daigaku

\section{Research article}

Keywords: limb salvage, amputation, patient-reported outcomes

Posted Date: June 7th, 2021

DOl: https://doi.org/10.21203/rs.3.rs-556756/v1

License: (c) (i) This work is licensed under a Creative Commons Attribution 4.0 International License. Read Full License 


\section{Abstract}

Background: Owing to advances in knowledge and technology, salvaging the limbs of patients with severe trauma and injuries is possible. However, severe limb injuries occasionally necessitate amputation because it allows patients to regain their social lives earlier than limb salvaging. Moreover, previous related investigations are retrospective cohort studies or meta-analyses of retrospective studies, and prospective cohort studies of patient-reported outcomes are extremely rare. This single-center, prospective cohort study aimed to compare the patient-reported outcomes at 1 year after injury between limb salvage and amputation and to elucidate whether amputation contributes to early recovery of functionality and quality of life.

Methods: We included 47 limbs of 45 patients with severe open fractures of the lower limb and categorized them into limb salvage and amputation groups. They were registered in the Database of Orthopedic Trauma by the Japanese Society for Fracture Repair at our center; data on patient-reported outcomes at 1 year after injury were obtained from this database. The mean patient age was 49.6 years. Patients' limbs were evaluated using the lower extremity functional scale (LEFS) and Short-Form 8 (SF-8). Early recovery was evaluated using functionality and quality-of-life questionnaires. Nonparametric statistical analyses were conducted.

Results: Of the 47 limbs, 34 limbs of 34 patients were salvaged and 13 limbs of 11 patients were amputated. Significant differences were noted between the limb salvage and amputation groups in terms of the LEFS scores (mean: 49.5 vs 33.1, $P=0.025$ ) and scores for the mental health component (mean: 48.7 vs 38.7, $\mathrm{P}=0.003$ ), role-physical component (mean: 42.2 vs $33.3, \mathrm{P}=0.026$ ), and mental component summary (mean: 48.2 vs $41.3, \mathrm{P}=0.042$ ) of the SF-8. The limb salvage group had better scores than the amputation group.

Conclusions: In this study, limb salvage results in better functional and mental health outcomes at 1 year after severe lower limb injury than after amputation. As reconstruction technology has advanced and limb salvaging has become possible, the focus of studies should now be based on the perspective of "how the patient feels"; hence, we believe that the results of this study, which is based on patient-reported outcomes, are meaningful.

\section{Background}

Advances in knowledge and technology have made salvaging the limbs of patients with severe trauma and injuries possible. However, amputation is occasionally the chosen course of action in severe limb injuries because it allows patients to regain their social lives earlier than limb salvaging [1-3]. Moreover, many previous investigations on this topic are retrospective cohort studies or meta-analyses on retrospective studies, whereas prospective cohort studies based on patient-reported outcomes are extremely rare. The present study aimed to compare the patient-reported outcomes, such as limb function and quality of life, at 1 year after injury between limb salvage and amputation in patients with Gustilo 
classification IIIb and IIIc fractures and to elucidate whether amputation leads to early recovery and regaining of social lives.

\section{Methods}

\section{Study design}

This single-center prospective cohort study was conducted at the Trauma and Reconstruction Center, Teikyo University Hospital, where patients with orthopedic injuries and trauma are managed. We used the following data obtained from the Database of Orthopaedic Trauma managed by the Japanese Society for Fracture Repair: patient age, sex, Gustilo classification, AO Foundation/Orthopedic Trauma Association (AO/OTA) classification, Orthopedic Trauma Association Open Fracture Classification (OTAOFC), OTA-OFC summative score [4], complications, preoperative and postoperative lower extremity functional scale (LEFS) score [5], postoperative Short-Form 8 (SF-8) score [6], number of operations, surgical site infection rate, and patient employment status.

\section{Participants}

Of the 439 limbs of 414 registered patients with open long-bone fractures at our center between February 2015 and December 2019, 69 limbs of 65 patients were diagnosed with Gustilo classification Illb and IIlc fractures, and 53 limbs of 51 patients were diagnosed with lower limb fractures, according to the AO/OTA classification $41-44$. This study included 47 limbs of 45 patients diagnosed as Gustilo classification Illb and IIIC as well as lower limb fractures (Fig. 1). The follow-up rate was $88.2 \%$. Patients were requested to provide self-reported outcomes at 9-15 months after injury. Salvaging or amputating the injured limb was decided by the facility for each patient depending on the extent of limb injury and the patient's general condition and social background. Of the 47 limbs of 45 patients, 34 limbs of 34 patients were salvaged, whereas 13 limbs of 11 patients were amputated. As an additional analysis with adjusted patient background, the same analysis procedures were carried out again after excluding patients with contralateral leg injuries, pelvic ring or acetabular fractures, and pre-injury functional impairment.

\section{Outcomes}

The primary outcomes of our study were patient-reported outcomes based on LEFS and SF-8 questionnaires $[5,6]$. The LEFS consists of 20 questions and is scored on a scale of $0-80$ points, with higher scores indicating higher functionality [5]. The SF-8 consists of 8 health dimensions and 2 summary measures and is calculated using a national average of 50 points (Norm-based Scoring), with higher scores indicating better health [6]. The secondary outcomes were the number of operations, surgical site infection rate, and patients' employment status (rate of change in employment), which are indicators of early recovery. The OTA-OFC summative score of the patients between the two groups were compared.

\section{Statistical analyses}


Statistical analyses were conducted using the John's Macintosh Project software (Version 15.1.0; SAS Institute, Cary, NC, USA). Non-parametric testing with significance levels defined by P-values of $<0.05$ was performed.

\section{Results}

Table 1 shows patient background characteristics, including the OTA-OFC summative score. Greater severity was observed in the amputation group than in the salvage group ( 14.3 and 9.8 , respectively; $P<$ 0.001). Figure 2 and Table 2 show the primary outcomes of the study. As shown in Fig. 2, the mean LEFS score was 49.5 (range, 15-80) for the salvage group and 33.1 (range, 5-61) for the amputation group, suggesting that the salvage group showed significantly superior primary outcomes compared with the amputation group ( $P=0.025$ ) (Table 2$)$. In the $S F-8$, significant differences were observed between the salvage and amputation groups in the mean scores of the role-physical component (42.2 and 33.3; $\mathrm{P}=$ 0.026 ), mental health (48.7 and 38.7; $P=0.003$ ), and mental component summary (48.2 and $41.3 ; \mathrm{P}=$ 0.042). The limb salvage group showed better outcomes than the amputation group in all aforementioned categories of the SF-8, but no significant differences were found between the two groups in the other categories of the SF-8 (Table 2).

Table 1

Background characteristics of the patients.

\begin{tabular}{|lll|}
\hline & Salvage & Amputation \\
\hline Patients (limbs*) & $34\left(34^{\star}\right)$ & $11\left(13^{\star}\right)$ \\
\hline Age (years) & $49.9(7-75)$ & $49.0(23-95)$ \\
\hline Sex (M: F) & $28: 6$ & $7: 4$ \\
\hline OTA-OFC score* & $9.8(6-14)$ & $14.3(8-15)$ \\
\hline Pre-injury LEFS & $79.4(66-80)$ & $72.7(22-80)$ \\
\hline $\begin{array}{l}\text { * Number of injured limbs. Data presented as mean (range). OTA-OFC summative score was } \\
\text { calculated for the number of injured limbs. }\end{array}$ \\
\hline
\end{tabular}


Table 2

Primary outcomes of the salvage and amputation groups

\begin{tabular}{|llll|}
\hline & Salvage & Amputation & P-values \\
\hline LEFS & $49.5(15-80)$ & $33.1(5-61)$ & $0.025^{*}$ \\
\hline SF-8 & & & \\
\hline Physical functioning & 41.8 & 34.6 & 0.076 \\
\hline Role physical & 42.2 & 33.3 & $0.026^{*}$ \\
\hline Bodily pain & 49.2 & 44.5 & 0.107 \\
\hline General health & 51.3 & 49.1 & 0.335 \\
\hline Vitality & 50.9 & 46.8 & 0.076 \\
\hline Social functioning & 44.8 & 38.5 & 0.060 \\
\hline Role emotional & 45.1 & 39.4 & 0.050 \\
\hline Mental health & 48.7 & 38.7 & $0.003 *$ \\
\hline Physical component summary & 43.8 & 39.0 & 0.073 \\
\hline Mental component summary & 48.2 & 41.3 & $0.042^{*}$ \\
\hline * indicates significant difference. Data presented as mean (range). \\
\hline
\end{tabular}

Table 3 shows the secondary outcomes of the study. Significant differences were noted in the number of operations between the salvage and amputation groups, with a mean of 4.4 (range, 2-8) and 2.3 (range, 1-6) $(P=0.001)$ operations, respectively. However, no significant differences in the other secondary outcomes were noted between the two groups.

Table 3

Secondary outcomes of the salvage and amputation groups

\begin{tabular}{|llll|}
\hline & Salvage & Amputation & P-values \\
\hline Number of operations & $4.4(2-8)$ & $2.3(1-6)$ & $0.001 *$ \\
\hline Infection rate (\%) & 35.3 & 38.5 & 1.000 \\
\hline Rate of change in employment (\%) & 26.5 & 18.2 & 0.705 \\
\hline
\end{tabular}

* indicates significant difference. Data presented as mean (range).

Additional analysis was conducted for the 29 limbs of 29 patients, after excluding 12 limbs of 10 patients with contralateral lower limb injury, 4 limbs of 4 patients with pelvic ring or acetabular fractures, and 2 limbs of 2 patients with pre-injury LEFS scores of $<77$ (Fig. 3). Hence, 24 patients in the salvage group 
and 5 patients in the amputation group were included in the additional analysis. The OTA-OFC summative scores were 10.1 and 14.6 for the salvage and amputation groups, respectively. Severity was greater in the amputation group than in the salvage group $(P=0.001)$ (Table 4). No significant differences in LEFS scores between the salvage and amputation groups were noted. However, for the SF-8, the scores of bodily pain component (salvage, 49.0 ; amputation, $38.7 ; \mathrm{P}=0.022$ ), vitality component (salvage, 51.7; amputation, $45.1 ; \mathrm{P}=0.036$ ), mental health component (salvage, 48.4; amputation, $36.3 ; \mathrm{P}=0.005$ ), and mental component summary (salvage, 48.2; amputation, 38.2; $P=0.012$ ) were significantly different between the two groups. The salvage group showed better scores in all aforementioned SF-8 categories than the amputation group (Table 5). No significant differences in secondary outcomes were noted between the two groups, except for the number of operations (salvage, 4.3 [range, 2-8]; amputation, 2.6 [range, 2-3]; $P=0.023$ ) (Table 6).

Table 4

Characteristics of the patients included in the additional analysis

\begin{tabular}{|lll|}
\hline & Salvage & Amputation \\
\hline Patients & 24 & 5 \\
\hline Age (years) & $48.6(7-73)$ & $37.4(23-47)$ \\
\hline Sex (M: F) & $21: 3$ & $4: 1$ \\
\hline OTA-OFC score & $10.1(7-14)$ & $14.6(14-15)$ \\
\hline Pre-injury LEFS & $79.8(78-80)$ & $80.0(80)$ \\
\hline
\end{tabular}

The additional analysis was performed after excluding patients with contralateral leg injuries, pelvic ring or acetabular fractures, and pre-injury LEFS score of $<77$. Data presented as mean (range). 
Table 5

Results of the additional analysis for the primary outcome

\begin{tabular}{|llll|}
\hline & Salvage & Amputation & P-values \\
\hline LEFS & $50.4(19-80)$ & $46.4(33-61)$ & 0.686 \\
\hline SF-8 & & & \\
\hline Physical functioning & 41.7 & 38.4 & 0.645 \\
\hline Role-physical & 42.2 & 35.7 & 0.296 \\
\hline Bodily pain & 49 & 38.7 & $0.022^{\star}$ \\
\hline General health & 51.9 & 46.3 & 0.085 \\
\hline Vitality & 51.7 & 45.1 & $0.036^{\star}$ \\
\hline Social functioning & 45 & 37.7 & 0.131 \\
\hline Role emotional & 44.9 & 36.9 & 0.073 \\
\hline Mental health & 48.4 & 36.3 & $0.005^{\star}$ \\
\hline Physical component summary & 44 & 39.9 & 0.194 \\
\hline Mental component summary & 48.2 & 38.2 & $0.012^{\star}$ \\
\hline
\end{tabular}

The additional analysis was performed after excluding patients with contralateral leg injuries, pelvic ring or acetabular fractures, and pre-injury LEFS scores of $<77$.

* indicates significant difference. Data presented as mean (range).

Table 6

Results of the additional analysis for secondary outcomes

\begin{tabular}{|lccc|}
\hline & Salvage & Amputation & P-values \\
\hline Number of operations & $4.3(2-8)$ & $2.6(2-3)$ & $0.023^{*}$ \\
\hline Infection rate (\%) & 41.6 & 40.0 & 0.945 \\
\hline Rate of change in employment (\%) & 16.7 & 0.0 & 0.200 \\
\hline $\begin{array}{l}\text { The additional analysis was performed after excluding patients with contralateral leg injuries, pelvic } \\
\text { ring or acetabular fractures, and pre-injury LEFS scores of < } 77 .\end{array}$ & \\
\hline * indicates significant difference. Data presented as mean (range). \\
\hline
\end{tabular}

\section{Discussion}


We initially believed that amputation contributed to early recovery of social life of patients with severe lower limb injuries. However, the obtained data on patient-reported outcomes at 1 year after injury, as investigated in this study, suggested that limb salvage provided better outcomes in terms of limb function and mental health. Additionally, while limb salvage required a higher number of operations, it did not affect the prevalence of surgical site infections and patient employment status. Furthermore, as shown in the LEFS score distribution in Fig. 2, we found that the LEFS score in the amputation group did not exceed 62. This finding implied that some functional restriction remained in the amputation group even in cases showing good progress (Fig. 2).

In our study, we noted cases of co-manifestation of injuries in other body parts owing to the severity of the primary injury. There were also patients who had existing pre-injury functional impairment caused by aging, which affected the evaluation. Therefore, we conducted an additional analysis using the same method, but for a limited number of patients, after excluding patients with contralateral leg injuries, pelvic ring or acetabular fractures, and a pre-injury LEFS score of $<77$ (Fig. 3). We established an LEFS score of $<77$ as the standard for pre-injury functional impairment because the median LEFS score in a study of healthy subjects was 77 [5]. Even after limiting the analysis to fewer patients and taking into account patient background, no differences were observed in the functional aspects of patient-reported outcomes at 1 year after injury, although the mental aspects of outcomes were better in the salvage group. However, considering the distribution of LEFS scores, while the amputation group did not perform well, some patients in the salvage group had poorer outcomes than all patients in the amputation group. In other words, not all patients in the salvage group had better outcomes than those in the amputation group (Fig. 4). In the case of unsuccessful limb salvage surgery, the salvage group had worse functional and mental outcomes than the amputation group [2]. Variations in the outcomes of the salvage group cannot be overlooked.

Several reports $[1-3,7,8]$ have suggested that amputation is a better approach for treating severe lower limb injuries than salvage. This claim is based on various perspectives of rebuttals against treatments that aim for limb salvage, such as advances in prosthetic limb technology, perioperative complications, duration of rehabilitation, number of operations, rate of operation site infections, and financial problems. Limb amputation is often the intervention of choice based on these reports. A study reported that limb salvage is accompanied by long rehabilitation, higher total cost, and an increased likelihood of a larger number of additional operations and re-admissions to the hospital [1]. Another study claimed that infection, reoperation, and hospitalization rates were significantly lower in the amputation group and that amputation provided better outcomes in terms of functioning and quality of life in patients with severe leg injuries [3]. New prosthetic limb technology allowed amputees to participate in activities and exercise and perform recreational activities that were previously impossible [7].

Some circumstances, such as those related to the patients' general condition and social background, necessitate amputation. However, according to several reports, limb salvage is more desirable than amputation in terms of psychological and long-term functional prognoses. By contrast, other reports have suggested that the time required by patients to return to work and hospitalization period are similar 
between the two interventions [1-3,9-11]. A meta-analysis showed that limb salvage and amputation are functionally equivalent, but limb salvage is the more psychologically acceptable approach [9]; this is consistent with another study that claimed amputation is more difficult to accept psychologically than salvage [3]. Most patients with salvaged limbs face problems in their daily lives owing to a limited range of motion, but none want amputation as an intervention [11]. A previous study showed no significant difference in the functional outcomes between the limb salvage and amputation groups for at least 7 years [1].

At present, it is becoming possible not only to preserve the injured limb but also to reconstruct it for better functional outcomes $[2,12-16]$. A study showed the increased possibility of limb salvage, even in patients with popliteal artery injuries [12], whereas another study reported that limb salvage is valuable even in patients with posterior tibial nerve injury [13]. Given these circumstances, the present study focused on early recovery based on the patients' perspectives.

One limitation of this study is the lack of randomization. According to the OTA-OFC summative score, patients who underwent limb amputation had more severe injuries. As amputation is ultimately chosen for mangled injuries, it is unavoidable for prospective studies. We believe that randomized studies involving a larger number of patients are required, either by excluding patients with high OTA-OFC summative scores or by taking OTA-OFC summative scores into account. Furthermore, to improve the postoperative outcome of severe leg injuries, it is essential to properly implement a comprehensive rehabilitation program that includes not only the physical aspects but also the psychological and social aspects [7]. At our facility, we believe that each patient was provided the opportunity to undergo rehabilitation with appropriate duration and quality, regardless of whether the intervention chosen was limb salvage or amputation.

With the advancement of reconstruction technology, it has become possible to salvage the affected limb. Therefore, it is crucial to understand the concept of "how the patient feels." We believe that the results of this study, which is based on patient-reported outcomes, are meaningful. For severe lower leg injuries, it is desirable to acquire knowledge and skills in revascularization, microvascular surgery, and flap surgery and consider salvaging the affected limb. However, the results of our additional analysis on a limited number of patients revealed that some patients with limb salvage had lower functional ability than patients with amputation and that limb salvage conducted without ascertaining the condition completely may result in worse outcomes than amputation.

\section{Conclusion}

We found that at 1 year after lower limb injury, limb salvage resulted in better functional and mental health outcomes than amputation. As reconstruction technology has advanced and limb salvaging has become possible, the focus of studies should now be based on the perspective of "how the patient feels"; hence, we believe that the results of this study, which is based on patient-reported outcomes, are meaningful. 


\section{Abbreviations}

AO/OTA

AO Foundation/Orthopedic Trauma Association

\section{LEFS}

lower extremity functional scale

OTA-OFC

Orthopedic Trauma Association Open Fracture Classifications

SF-8

Short-Form 8

\section{Declarations}

Ethics approval and consent to participate: The study conformed to the principles of the Declaration of Helsinki and its amendments. Written and verbal informed consent was obtained from all patients prior to undergoing treatment and participating in this study. This study was approved by the Institutional Review Board of Teikyo University Ethical Review Board for Medical and Health Research Involving Human Subjects (approval number: 14-167-3).

Consent for publication: Not applicable.

Availability of data and materials: All datasets used and/or analyzed during the current study are available in the Database of Orthopaedic Trauma managed by the Japanese Society for Fracture Repair (DOTJ).

Competing interests: The authors declare that they have no competing interest.

Funding: This research received no specific grant from any funding agency in the public, commercial, or not-for-profit sectors.

Authors' contributions: $\mathrm{YN}, \mathrm{AF}, \mathrm{KM}, \mathrm{KI}$, and TS assisted in data management and collection. $\mathrm{TI}$ and $\mathrm{YW}$ analyzed and interpreted the patient data. All authors have read and approved the final manuscript.

Acknowledgments: This manuscript was originally published in the journal Kossetsu $(2021 ; 43: 123-127)$ in Japanese. It is reprinted here in English with the permission of the Japanese Society for Fracture Repair (JSFR).

\section{References}

1. Busse JW, Jacobs CL, Swiontkowski MF, Bosse MJ, Bhandari M, Evidence-Based Orthopaedic Trauma Working Group. Complex limb salvage or early amputation for severe lower-limb injury: A meta-analysis of observational studies. J Orthop Trauma. 2007;21:70-6. 
2. Schirò GR, Sessa S, Piccioli A, Maccauro G. Primary amputation vs limb salvage in mangled extremity: A systematic review of the current scoring system. BMC Musculoskelet Disord. 2015;16:372.

3. Fioravanti M, Maman P, Curvale G, Rochwerger A, Mattei JC. Amputation versus conservative treatment in severe open lower-limb fracture: A functional and quality-of-life study. Orthop Traumatol Surg Res. 2018;104:277-81.

4. Hao J, Cuellar DO, Herbert B, Kim JW, Chadayammuri V, Casemyr N, et al. Does the OTA open fracture classification predict the need for limb amputation? A retrospective observational cohort study on 512 patients. J Orthop Trauma. 2016;30:194-9.

5. Dingemans SA, Kleipool SC, Mulders MAM, Winkelhagen J, Schep NWL, Goslings JC, et al. Normative data for the lower extremity functional scale (LEFS). Acta Orthop. 2017;88:422-6.

6. Tokuda Y, Okubo T, Ohde S, Jacobs J, Takahashi O, Omata F, et al. Assessing Items on the SF-8 Japanese Version for Health-Related Quality of Life: A Psychometric Analysis Based on the Nominal Categories Model of Item Response Theory. Value Health. 2009;12:568-73.

7. Shawen SB, Keeling JJ, Branstetter J, Kirk KL, Ficke JR. The mangled foot and leg: Salvage versus amputation. Foot Ankle Clin. 2010;15:63-75.

8. Perkins ZB, Yet B, Glasgow S, Cole E, Marsh W, Brohi K, et al. Meta-analysis of prognostic factors for amputation following surgical repair of lower extremity vascular trauma. Br J Surg. 2015;102:43650.

9. Akula M, Gella S, Shaw CJ, McShane P, Mohsen AM. A meta-analysis of amputation versus limb salvage in mangled lower limb injuries - The patient perspective. Injury. 2011;42:1194-7.

10. Christensen J, Ipsen T, Doherty P, Langberg H. Physical and social factors determining quality of life for veterans with lower-limb amputation(s): A systematic review. Disabil Rehabil. 2016;38:2345-53.

11. Tunali O, Saglam Y, Balci HI, Kochai A, Sahbaz NA, Sayin OA, et al. Gustilo type IIIC open tibia fractures with vascular repair: Minimum 2-year follow-up. Eur J Trauma Emerg Surg. 2017;43:50512.

12. Dua A, Desai SS, Shah JO, Lasky RE, Charlton-Ouw KM, Azizzadeh A, et al. Outcome predictors of limb salvage in traumatic popliteal artery injury. Ann Vasc Surg. 2014;28:108-14.

13. Momoh AO, Kumaran S, Lyons D, Venkatramani H, Ramkumar S, Chung KC, et al. An argument for salvage in severe lower extremity trauma with posterior tibial nerve injury: The Ganga Hospital experience. Plast Reconstr Surg. 2015;136:1337-52.

14. Loja MN, Sammann A, DuBose J, Li CS, Liu Y, Savage S, et al. The mangled extremity score and amputation: Time for a revision. Trauma Acute Care Surg. 2017;82:518-23.

15. Frisvoll C, Clarke-Jenssen J, Madsen JE, Flugsrud G, Frihagen F, Andreassen GS, et al. Long-term outcomes after high-energy open tibial fractures: Is a salvaged limb superior to prosthesis in terms of physical function and quality of life? Eur J Orthop Surg Traumatol. 2019;29:899-906.

16. Hohenberger GM, Konstantiniuk P, Cambiaso-Daniel J, Matzi V, Schwarz AM, Lumenta DB, et al. The mangled extremity severity score fails to be a good predictor for secondary limb amputation after 
Figures

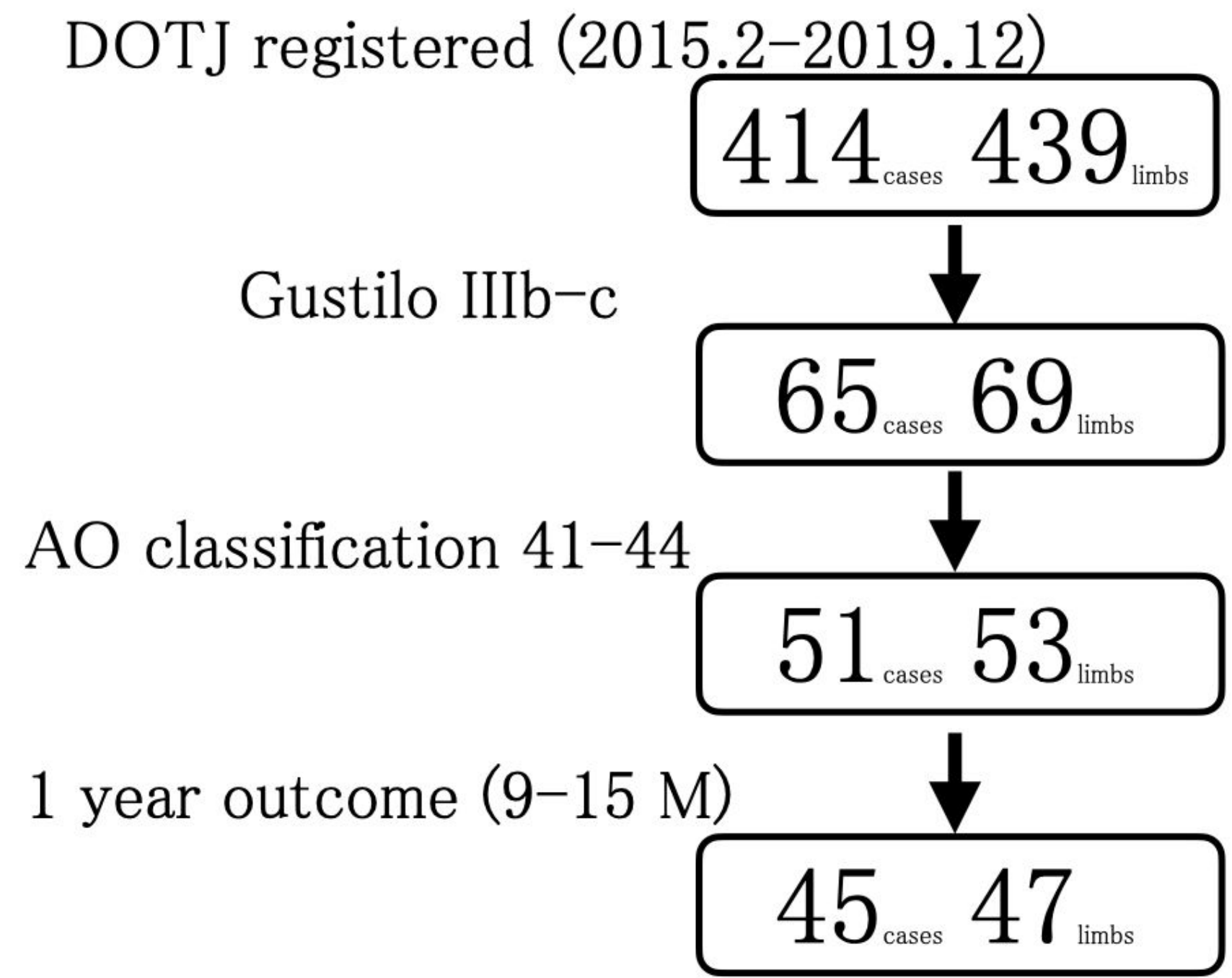

Figure 1

Flow diagram of the study design. 
LEFS

80

60

40

8

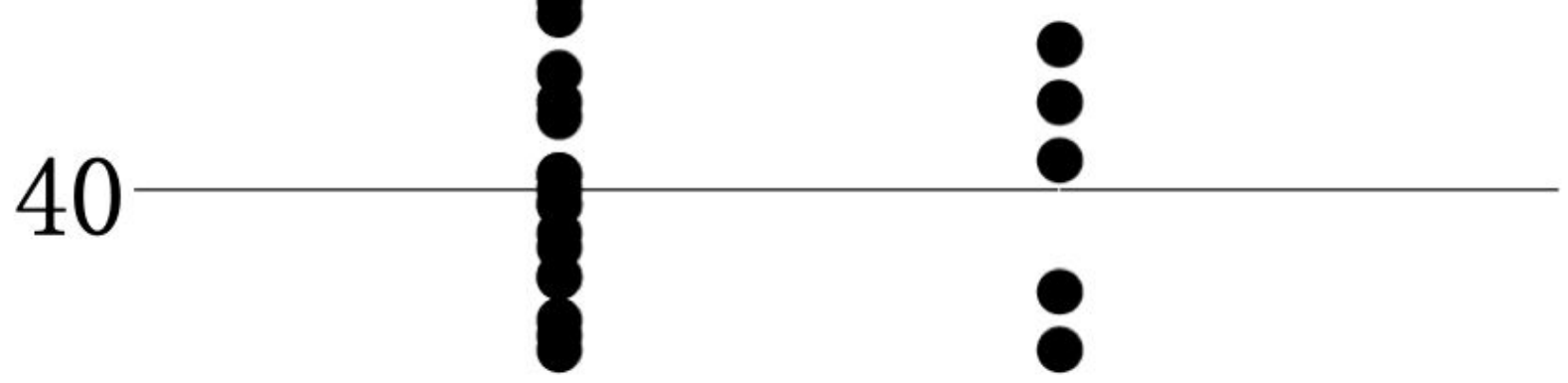

20

0

0

8

\section{Salvage Amputation}

Figure 2

Distribution of the lower extremity functional scale score of the enrolled patients $\rrbracket$ 
Patients

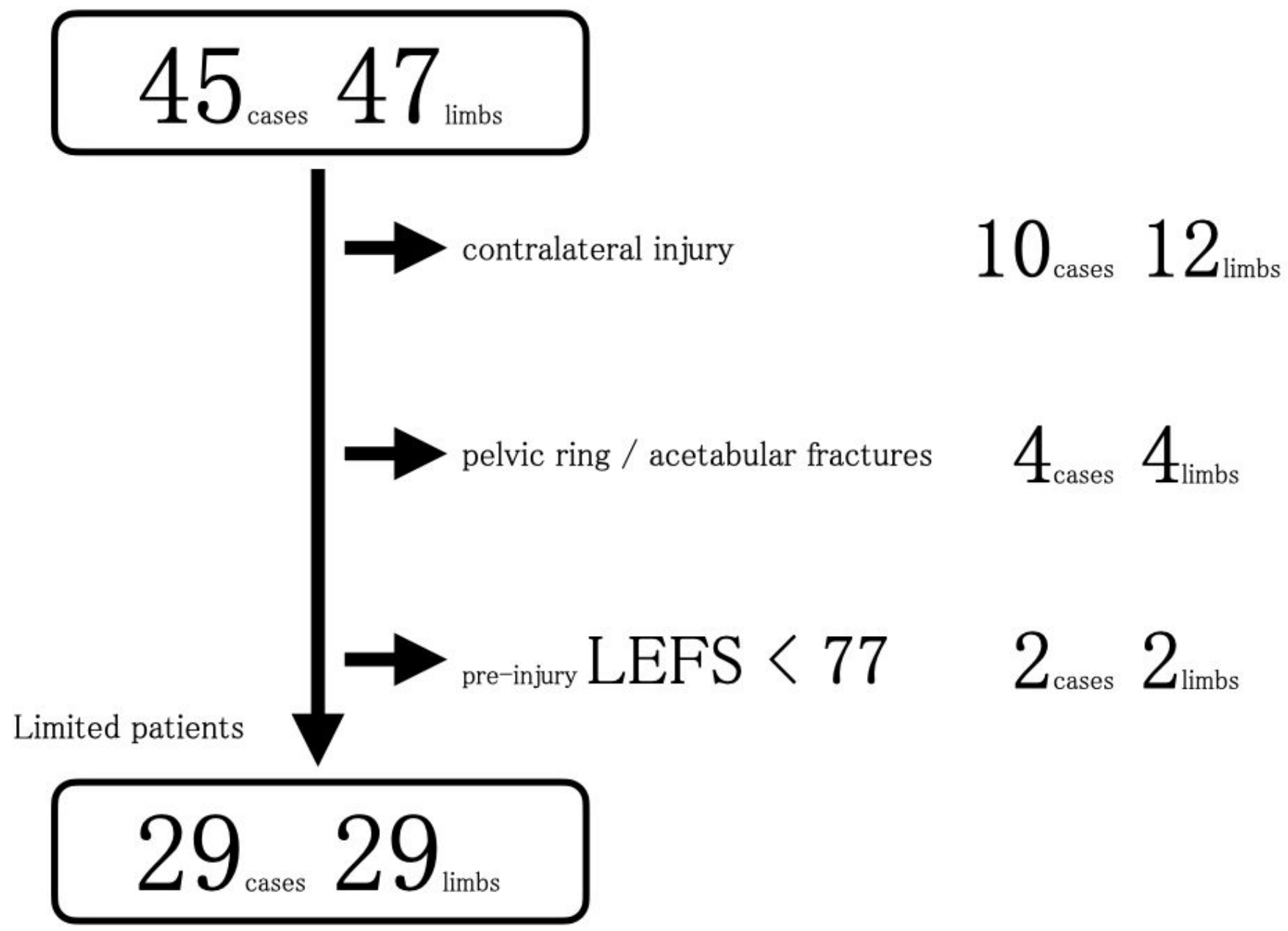

Figure 3

Flow diagram of the additional analysis. 
LEFS
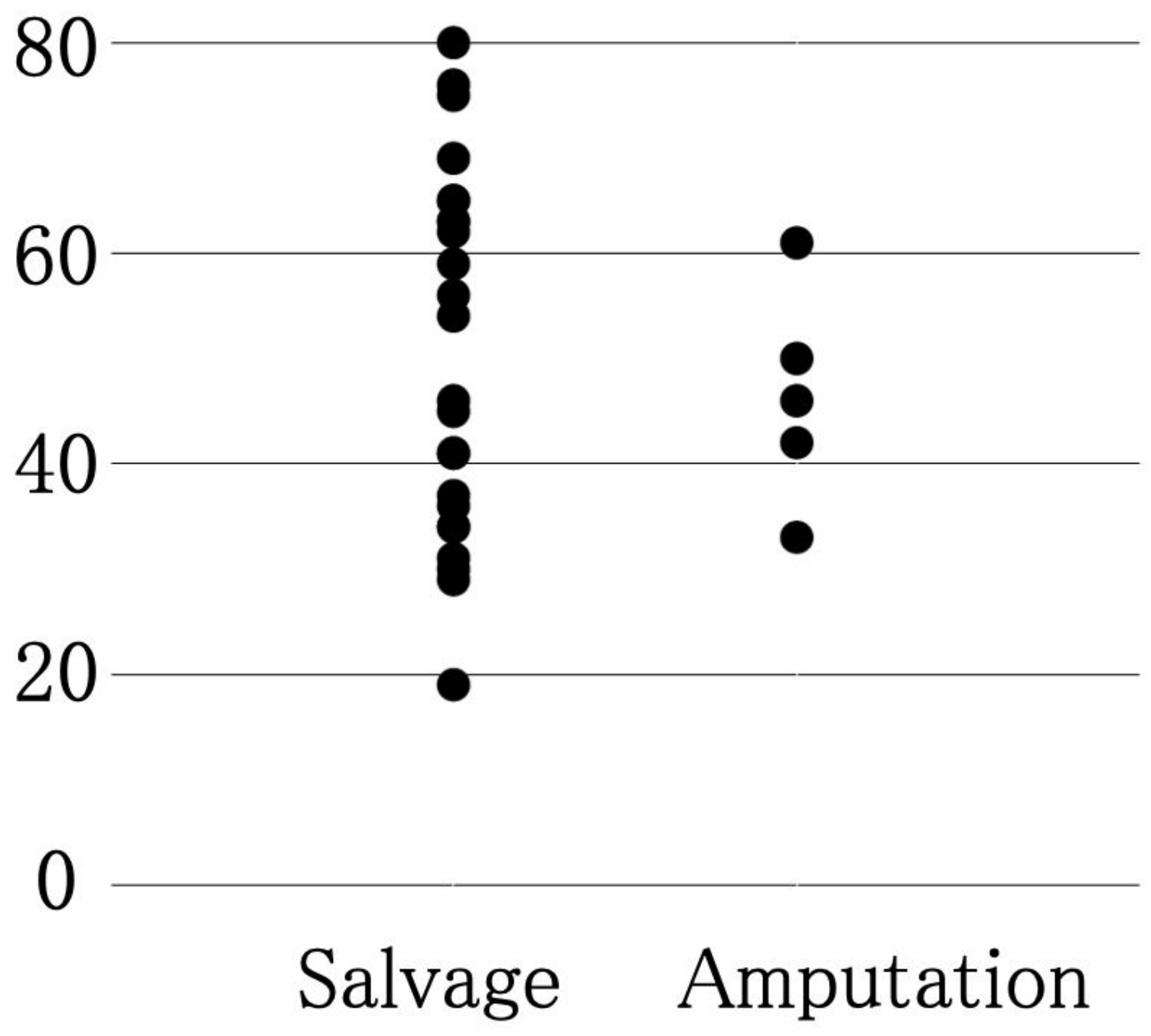

Figure 4

Additional analysis of the lower extremity functional scale (LEFS) scores in a limited number of patients, after excluding those with contralateral leg injuries, pelvic ring or acetabular fractures, and a pre-injury LEFS score of $<77$. 